\title{
OPERA-reassessing data on the energy dependence of the speed of neutrinos
}

\author{
Giovanni AMELINO-CAMELIA, ${ }^{1,2}$ Giulia GUBITOSI, ${ }^{3}$ Niccoló LORET,,${ }^{1,2}$ \\ Flavio MERCATI,${ }^{4}$ Giacomo ROSATI,${ }^{1,2}$ and Paolo LIPARI ${ }^{2}$ \\ ${ }^{1}$ Dipartimento di Fisica, Università di Roma "La Sapienza", P.le A. Moro 2, 00185 Roma, EU \\ ${ }^{2}$ INFN, Sez. Romal, P.le A. Moro 2, 00185 Roma, EU \\ ${ }^{3}$ Department of Physics, University of California, Berkeley, CA 94720, USA \\ ${ }^{4}$ Departamento de Física Téorica, Universidad de Zaragoza, Zaragoza 50009, Spain
}

\begin{abstract}
We offer a preliminary exploration of the two sides of the challenge provided by the recent OPERA data on superluminal neutrinos. On one side we stress that some aspects of this result are puzzling even from the perspective of the wild quantum-gravity literature, where arguments in favor of the possibility of superluminal propagation have been presented, but not considering the possibility of such a sizeable effect for neutrinos of such low energies. We feel this must encourage particularly severe scrutiny of the OPERA result. On the other side, we notice that the OPERA result is reasonably consistent with $\mu$-neutrino-speed data previously obtained at FERMILAB, reported in papers of 2007 and 1979. And it is intriguing that these FERMILAB79 and FERMILAB07 results, when combined with the new OPERA result, in principle provide a window on $\mu$-neutrino speeds at different energies broad enough to compare alternative phenomenological models. We test the discriminating power of such an approach by using as illustrative examples the case of special-relativistic tachyons, the case of "Coleman-Glashow-type" momentum-independent violations of the special-relativistic speed law, and the cases of linear and quadratic energy dependence of the speed of ultrarelativistic muon neutrinos. Even just using $\mu$-neutrino data in the range from $\sim 3 \mathrm{GeVs}$ to $\sim 200 \mathrm{GeVs}$ the special-relativistic tachyon and the quadratic-dependence case are clearly disfavoured. The linear-dependence case gives a marginally consistent picture and the Coleman-Glashow scenario fits robustly the data. We also comment on Supernova 1987a and its relevance for consideration of other neutrino species, also in relation with some scenarios that appeared in the large-extra-dimension literature.
\end{abstract}

\section{PRELIMINARIES AND MOTIVATION}

The OPERA collaboration recently reported [1] evidence of superluminal behavior for $\mu$ neutrinos $\left(\nu_{\mu}\right)$. Specifically, these "OPERA/CNGS11 data" (data reported by the OPERA collaboration, CNGS beam, in 2011 [1]) imply for the speed of such neutrinos the estimate

$$
v_{\nu_{\mu}}-1=(2.48 \pm 0.28 \text { (stat) } \pm 0.30(\text { sys })) \cdot 10^{-5},(1)
$$

a significance of six standard deviations (we use units such that the speed of light is $c=1$ ).

This would be the most significant discovery in fundamental physics over the last several decades, so the OPERA data will inevitably motivate a healthy exploration of both possible outcomes: on one side the data, particularly the possibility of unnoticed systematic biases, should be scrutinized very carefully, and on the other side, which however will require (also in light of some of the considerations we here offer) the guidance of some dedicated model building, one may look for corroborating evidence in totally independent measurements, at different energies using different techniques.

We here report a preliminary exploration of these two sides. We are going to provide further motivation for careful scrutiny of the data by observing that such a result cannot be accommodated in any reasonably well studied existing theory speculation. In particular, even in the quantum-gravity literature, small parts of which have provided motivation for searches of violations of Lorentz symmetry, including some proposals of superluminal type, one finds no scenario with an effect of that magnitude for particles with energies so far from the Planck scale.

For the opposite side, the one of attempts to find evidence corroborating the OPERA/CNGS11 result, also exploiting the guidance of some dedicated phenomenological models, our main message is that combining CNGS11 with other previously-obtained data on the speed of $\mu$-neutrinos we might have sufficient guidance to filter significantly the list of candidate phenomenological models. We find that a particularly interesting picture is obtained when combining the CNGS11 data with the "FERMILAB07" data (obtained at Fermilab, by the MINOS collaboration, and reported in $2007[2]$ ) and the "FERMILAB79" data (also obtained at Fermilab, and reported in 1979 [3]). This allows us to look at speed of $\mu$-neutrinos with data populating with acceptable density the range from $\sim 3 \mathrm{GeV}$ to $\sim 200 \mathrm{GeV}$. So if one takes these data at face value (as a working assumption, looking for evidence possibly corroborating CGNS11) one has a criterion to select phenomenological models, whose guidance could be used to set up particularly meaningful other tests of the superluminal $\mu$-neutrino hypothesis.

Mostly as a way to test that our proposal of combining the new CGNS11 data with the previous FERMILAB07 and FERMILAB79 data actually can "discriminate models" (though only conditionally on the working assumption that the data can be taken at face value), we focus on a few very simple phenomenological pictures of superluminal particles: a "standard" (imaginary-mass) special-relativistic tachyon, the case of "Coleman-Glashow neutrinos" [4, 5], with real mass and violatons of the special-relativistic speed law which are momentum independent in the ultrarelativistic regime, and two "DSR-type pictures" [6-10], with real mass, possibly "deformations" (rather than preferred-frame breakdowns) of Lorentz symmetry, and momentum-dependent speed in the ultrarelativistic regime. We expose some discriminating power for the strategy here proposed by finding that, among these illustrative examples of phenomenological models, the special-relativistic-tachyon scenario and the DSR model with quadratic dependence on the deformation scale are clearly 
disfavored by CGNS11+FERMILAB07+FERMILAB79 data, while, at least adopting a conservative approach, the ColemanGlashow scenario and the DSR model with linear dependence give a reasonably good description. We also comment on Supernova 1987a and its relevance for consideration of other neutrino species, also in relation with some scenarios that appeared in the large-extra-dimension literature.

\section{NO QUANTUM-GRAVITY MODELS, BUT SOME SIMPLE PHENOMENOLOGICAL TEST THEORIES}

The physics literature provides no compelling argument for special-relativistic tachyons and only some rare instances where departures from standard Lorentz symmetry could be motivated. We feel that the most compelling arguments for possible departures from standard Lorentz symmetry are found in the part of the quantum gravity literature which motivates [11-14] the adoption of a nonclassical-geometry description of spacetime, with associated violations or deformations of Lorentz symmetry. Moving from the level of "theories" to the one of "phenomenological pictures" a noteworthy possibility is the much-studied idea of large extra dimensions, within which several authors have motivated mechanisms for violations of Lorentz symmetry (see, e.g., Refs. [15-19] and references therein).

We actually postpone a detailed discussion of the relevant quantum-gravity literature to a forthcoming companion paper [20], since it would impose here a lengthy aside. We do note here that superluminal particles have been motivated in parts of the quantum-gravity literature, and aspects of the quantum-gravity problem offer solid motivation for rather strong particle dependence of the effects (so that it would not be surprising to find the superluminal behavior of neutrinos, possibly even just some types of neutrinos, to be a few orders of magnitude stronger than for other particles). But the bottom line is that [20] the effect reported by OPERA/CGNS11 appears to be much too strong to be of quantum-gravity origin: one could rather compellingly motivate from quantum gravity the qualitative nature of that experimental result but the magnitude appears to be unbelievably gigantic by quantum-gravity standards.

The OPERA result is in the peculiar position not only of conflicting with one of the cornerstones of current theories, but also of conflicting with the most appealing alternatives. We are fully aware of the fact that some major discoveries in the history of physics shared this fate, but nonetheless we feel this must be viewed as an invitation to be cautious with theory speculations inspired by the OPERA result, and also as an invitation to scrutinize the result meticulously.

Going back to the issue of the puzzling magnitude of the OPERA effect, as seen from a quantum-gravity perspective, let us here be satisfied to discuss it specifically within (two versions of) one of the pictures considered in the quantumgravity literature, a picture from which we shall also borrow inspiration for two of the test theories here used as illustrative examples of "pure-phenomenology interest". Let us start this off with the dispersion/on-shellness relation

$$
E^{2}=m^{2}+p^{2}+\ell_{1} E p^{2},
$$

which has been much studied in parts of the quantum-gravity literature. It is a scheme with particles of ordinary real mass $\left(m^{2}>0\right)$, but it is superluminal, as easily verified computing the speed $d E / d p$ in the ultrarelativistic regime $E \gg m$ of (2) (for positive $\ell_{1}$ ). Evidently such a dispersion relation cannot be accommodated within standard special-relativistic Lorentz symmetry, but interestingly it is possible to implement this dispersion relation in a deformed-Lorentz-symmetry framework, the so called "DSR" framework [6-10], without any breakdown of the relativity of reference frames: in such DSR frameworks the laws of transformation between inertial observers are $\ell_{1}$-modified, but the equivalence of all inertial observers is preserved. A few hundred papers have been devoted to this possibility, within the quantum-gravity literature, over the last decade, but all of them assume that the deformation scale $\ell_{1}$ should be of the order of the "Planck length", i.e. $\left(10^{19} \mathrm{GeV}\right)^{-1}$, or at best (and only if confined to spin- $-1 / 2$ particles) a few orders of magnitude greater than that. The Planck length is the only natural scale of the quantum gravity problem, but if we use (2) with $\ell_{1}$ of the order of the Planck length then the conclusion would be for OPERA neutrinos to be affected only at the level of 1 part in $10^{18}$, rather than 1 part in $10^{5}$ : the qualitative feature is not completely foreign to the quantum-gravity literature but the magnitude of the effect is off by 13 orders of magnitude. Another way to characterize the situation equivalently is to state that the OPERA data appear to probe, from the viewpoint of (2), a scale of $\ell_{1} \sim\left(10^{6} \mathrm{GeV}\right)^{-1}$, which is a small scale by the standards of other areas of physics but is a gigantically macroscopic length scale by the standards of quantum-gravity researchers.

One easily sees that this point applies also to the similar case of a quadratic formula

$$
E^{2}=m^{2}+p^{2}+\ell_{2}^{2} E^{2} p^{2}
$$

which also has been at the center of strong interest in parts of the recent quantum-gravity literature. For this sort of quadratic corrections the OPERA/CNGS11 neutrinos would be probing the even more gigantic length scale of $\ell_{2} \sim$ $\left(10^{3} \mathrm{GeV}\right)^{-1}, 16$ orders of magnitude bigger than the Planck length!!

We shall not dwell on this any further here. We shall be satisfied to have contributed a bit to the first assessments of how surprising this result must be considered. We feel it would be legitimate to argue that the OPERA result is so puzzling that it should be completely ignored until it somehow gets confirmed independently. But, as anticipated above, we here take the attitude that the OPERA result itself, when combined with other available results on the speed of $\mu$ neutrinos, can provide guidance for attempts of confirming its result truly independently, in other energy regimes, using different experimental techniques. And we expect that the development of suitable test theories could play a crucial role for guiding the setup of any such attempts.

We illustrate here how we envisage this interplay between available data and test theories, by considering a few rudimentary test theories of the energy dependence of the speed of $\mu$ neutrinos and assessing on them the discriminating power of our approach.

Our first candidate is the "standard" special-relativistic tachyon, which in the ultrarelativistic regime has speed

$$
v=\sqrt{1+\frac{\mathcal{M}^{2}}{E^{2}}} \simeq 1+\frac{1}{2} \frac{\mathcal{M}^{2}}{E^{2}}
$$


where for convenience we introduced the parameter $\mathcal{M}^{2}$ which is positive (the opposite, $\mathcal{M}^{2}=-m^{2}$, of the square of the imaginary mass $m$ of a special-relativistic tachyon).

Our second candidate is inspired by work of Coleman and Glashow [4, 5] which would lead to the following description of the dependence of speed on energy in the ultrarelativistic regime

$$
v \simeq 1-\frac{1}{2} \frac{m^{2}}{E^{2}}+\delta
$$

where $\delta$ is the parameter characterizing the maximum attainable speed by the particle. Evidently this maximum attainable speed may be greater than $c$, if $\delta$ is positive, and yet also (5) assumes [4, 5] the mass $m$ to be real. This Coleman-Glashow picture evidently requires departures from the special-relativistic description of Lorentz symmetry. And there are no established results on possibly implementing this as a "deformation" of Lorentz symmetry, so it has been studied exclusively as a scenario for a full "breakdown" of Lorentz symmetry, including the emergence of a preferred frame. But it is a much studied framework, which has shown some robustness (for what concerns logical consistency) in several attempted applications, and can be even accommodated naturally within the more general framework of "Standard Model Extension" [21, 22].

For our third and fourth candidates we go back to the DSRtype scenarios already briefly mentioned above, for Eqs. (2) and (3). Evidently the OPERA/CNGS11 result could not fit within the original spirit of studies of these scenarios, since as mentioned the effects are much larger than expected. But, as we are looking for candidates to test the "phenomenology content" of data so far available on $\mu$ neutrinos, we felt having some DSR-type candidates could be valuable, at least for illustrating the idea of departures from special relativity which do not require introducing a preferred frame. So for the third scenario we take, from (2), the following description of the dependence of speed on energy in the ultrarelativistic regime 1

$$
v \simeq 1-\frac{1}{2} \frac{m^{2}}{E^{2}}+\ell_{1} E
$$

where $\ell_{1}$ is the parameter characterizing the dependence (in this case linear) of the speed of ultrarelativistic particles on momentum. While evidently the proposal of this DSR scheme [6, 7] had in mind that $\ell_{1}$ would be at least roughly of the order of the Planck length, we shall here allow it, for the sake of the argument, to be even much greater than that.

\footnotetext{
1 The same formula for the dependence of speed on energy can of course also be introduced [11 23 [24] in scenarios where Lorentz symmetry is fully broken, with a preferred frame. Actually the first study that brought this proposal to the attention of the quantum-gravity community, the one in Ref. [11], assumed broken Lorentz symmetry, and only later it was realized that a DSR-type formulation was also possible [6 7]. We here label these formulas as "DSR-type" because it is relevant for the (however limited) representativity of the small sample of scenarios we consider that two of the scenarios are also known to be compatible with the Principle of Relativity of inertial frames ("only" at the cost of adopting DSR-type [6, 7] deformed laws of transformation between inertial observers).
}

Similarly we take inspiration from the quantum-gravity interest in Eq. (3), to consider here (once again far away from the originally intended, Planckian, range of scales) the phenomenology of

$$
v \simeq 1-\frac{1}{2} \frac{m^{2}}{E^{2}}+\ell_{2}^{2} E^{2}
$$

where the parameter $\ell_{2}$ evidently charaterizes the quadratic dependence of the speed of ultrarelativistic particles on momentum.

A noteworthy observation is that the large-extra-dimension literature has provided some arguments motivating a superluminal behaviour of neutrinos, but confined to some range of scales. Staring at the OPERA result it is in particular striking to look back at papers such as Refs. [15, 16], which more than a decade ago, argued for superluminal behaviour of neutrinos with onset at a scale not far from the electroweak scale. And more recently (but much before the OPERA study) other large-extra-dimension investigations have reported the possibility of superluminal behaviour for neutrinos, confined to specific ranges of energy, as discussed in particular in Refs. [18, 19] (also see Ref. [25] for related phenomenology work in preparation for OPERA). This literature appears not to provide us with a clear candidate test theory that would be relevant here for our purposes, but we shall take into account its indications when, in later parts of this manuscript, we move away from our main focus on $\mu$ neutrinos in the $3-200 \mathrm{GeV}$ range, and consider briefly the behaviour of other species of neutrinos in other ranges of energy.

\section{OPERA+FERMILAB07}

First reactions to the OPERA/CNGS11 result are of great astonishment. From the theory perspective we fully ascribe, as stressed above, to that sort of reaction. But on the other hand we feel there is too little awareness of the fact that, if one looks exclusively at previous experimental results on the speed of $\mu$ neutrinos, this OPERA result does not really "come out of the blue". We shall here show that the CNGS11 result actually fits rather naturally with the "FERMILAB07" data (obtained at Fermilab, by the MINOS collaborations, and reported in 2007 [2]) and with the "FERMILAB79" data (also obtained at Fermilab, and reported in 1979 [3]).

In this short section let us just compare the OPERA/CNGS11 result with the FERMILAB07 result reported in Ref. [2]:

$$
v-1=(5.1 \pm 2.9) \cdot 10^{-5}
$$

for $\mu$ neutrinos ${ }^{2}$ of $\sim 3 G e V$.

This previous FERMILAB07 result (while intrinsically having only a significance of less than two standard deviations) goes in the same direction as the new CNGS11 result,

\footnotetext{
${ }^{2}$ Because of the exploratory goals of our analysis we shall not dwell much on the details of the composition of the data. If we label a result as a $\mu$ neutrino result it simply means we expect the contamination from other neutrino species to be "small".
} 
and the two results appear to be rather naturally compatible with each other. Actually, they are not only compatible but they were also determined at relatively close energies, and this for our purposes is not welcome: if one only combines the OPERA/CNGS11 result and the FERMILAB07 result one gets very limited ability to discriminate between models predicting different forms of energy dependence.

\section{OPERA+FERMILAB07+FERMILAB79}

It must be evident at this point that our main interest is in the form of energy dependence of the speed of neutrinos (actually primarily $\mu$ neutrino, see later). This is also reflected in the choice of illustrative examples of test theories on which we focus: they are simple and representative of significant classes of related speculations, and they also represent alternative options for how the speed of ultrarelativistic neutrinos could depend on energy. And it is also evident that because of these objectives it is desirable for us to consider data at relatively high energies, at least somewhat higher that the range covered by OPERA/CGNS11 and MINOS/FERMILAB07. From this perspective it is interesting to reconsider the FERMILAB79 data on the speed of neutrinos reported in 1979 in Ref. [3]. These extend all the way from $\sim 30 \mathrm{GeV}$ to $\sim 200 \mathrm{GeV}$, so they open a very valuable window for our purposes.

The energy dependence is so crucial for our purposes that we shall here not use OPERA's most significant result, (1), obtained by combining CC-internal and external events [1], since it carries no verification of the neutrino energies. We find most valuable for our purposes to consider only the $\mathrm{CC}$ internal events for which [1]

$$
\begin{aligned}
& \left.\left(v_{\nu_{\mu}}-1\right)\right|_{14 G e V}=(2.18 \pm 0.77(\text { stat }) \pm 0.30(\text { sys })) \cdot 10^{-5} \\
& \left.\left(v_{\nu_{\mu}}-1\right)\right|_{43 G e V}=(2.75 \pm 0.75(\text { stat }) \pm 0.30(\text { sys })) \cdot 10^{-5}
\end{aligned}
$$

These are of lower significance than (1), but carry the energy information crucial for our purposes.

In our Fig. 1 we show the neutrino data ${ }^{3}$ from Fig. 3 of Ref. [3], together with the MINOS/FERMILAB07 result, and the OPERA results we just noted.

Concerning the FERMILAB79 data we should stress that they actually concern the difference between the speed of the $\mu$-neutrino and the speed of muons, but we shall be not too embarrassed of taking as working assumption of this first exploratory study that the speed of muons, at least in that range of energies, is faithfully described by standard special relativity, so we shall handle the FERMILAB79 data as determinations of the speed of the $\mu$-neutrino at various neutrino energies. More concerning for us is the fact that the analysis in Ref. [3] relies very significantly on correcting for a large bias: it was realized [3] that one should correct for the fact that the relevant muons taking part in that differential measurement ended up being on paths that were effectively longer

\footnotetext{
${ }^{3}$ We here do not consider the antineutrino data also found in Ref. [3]. we feel in this exploratory stage it is an asset to look exclusively at $\mu$-neutrino data.
}

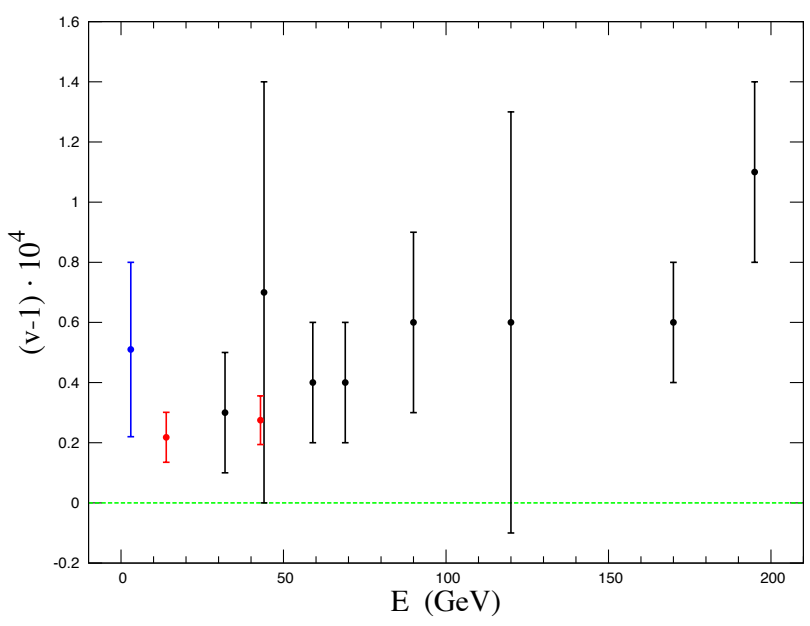

FIG. 1. The results for the speed of $\mu$ neutrinos reported by OPERA (red), MINOS (blue) and in Fig. 3 of Ref. [3] (black).

than the path of the neutrinos they were "racing" against. The authors of Ref. [3] conclude that this would effectively produce a rigid (equal at all energies) downward shift of all estimates of the neutrino velocity, and that a very sizable such downward shift should be applied. Specifically this bias correction, which we shall denote with $b_{1979}$, was estimated [3] at $b_{1979}=\left(0.5_{-0.1}^{+0.2}\right) \cdot 10^{-4}$. Our Fig. 2 shows the effect of $b_{1979}=0.5 \cdot 10^{-4}$ on the black points of Fig. 1 . As seen comparing Figs. 1 and 2 the nest result on the findings of Ref. [3] roughly amounts to the subtraction of a large estimated "background", leaving the analysis with a small "signal".

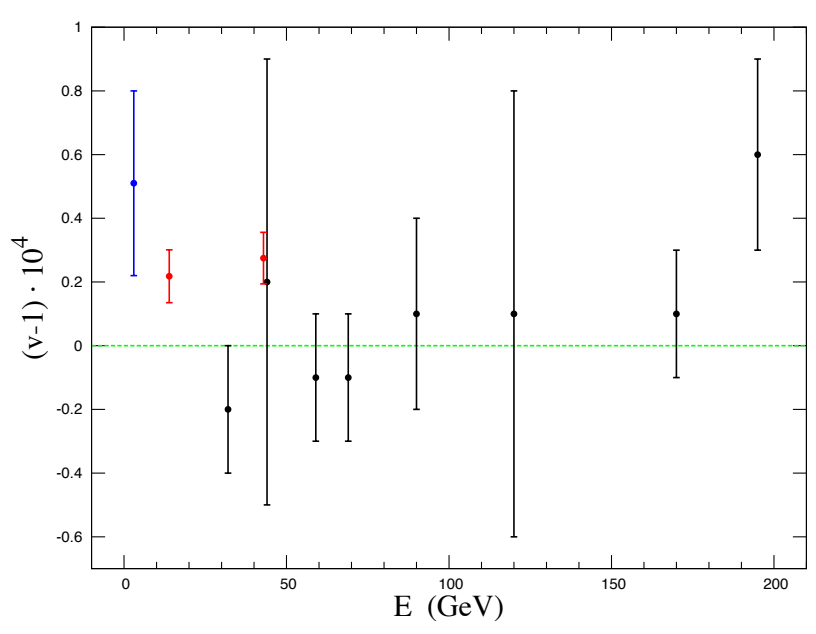

FIG. 2. As in fig.1 but taking into account the large bias correction discussed at the end of Ref. [3].

Looking at our Fig. 1 and even our Fig. 2 one cannot fail to notice that, while surely previous measurements had not reached enough significance to make substantial claims, the overall picture is perfectly consistent with what was then very recently reported by OPERA. And concerning the issue of a 
possible energy dependence of the speed of ultrarelativistic muon neutrinos we feel that it deserves at least some mention that Ref. [3] reported an estimate of the dependence on energy of the $\mu$ neutrinos, with linear law and slope of $(0.3 \pm 0.1) \cdot 10^{-6}$, so an indication of non-vanishing slope (which is visible in the summary of Ref. [3] data in our Figs. 1 and 2) with a significance of three standard deviation. This went largely unnoticed but we feel that in light of the results reported by OPERA it may require to be reconsidered.

One of our main points in this section is that the data of Ref. [3], with information at different energies, may be a valuable resource for attempts of interpretation of the OPERA result. We shall do this in the following, while proceeding cautiously because of the concerns for the mentioned large bias estimate given in Ref. [3] for their data.

\section{A. OPERA+FERMILAB07+FERMILAB79 and non-tachyonic special relativity}

It is easy to see that the comparison of standard nontachyonic special relativity with the black and blue (preOPERA) data points of our Fig. 1 could have produced some serious concerns. But using the data of Ref. [3], bringing in the mentioned large bias correction, the situation is the one of the black and blue points in Fig. 2, which is reasonably consistent with non-tachyonic special relativity. The assumed pre-OPERA starting point was our Fig. 2, so non-tachyonic special relativity was considered to be in good health.

But non-tachyonic special relativity definitely is no longer in good health if one takes at face value the data very recently reported by OPERA/CNGS11 data [1]. This is evident from the higher-significance OPERA result here reported in Eq. (1), but it is important for our purposes to establish that one can conclude that non-tachyonic special relativity is disfavored even only using the lower-significance OPERA data (but with sharper information on the energy of the neutrinos) which we are using.

To render this claim fully robust we first notice that the reduced $\chi^{2}$ of a fit of non-tachyonic-special-relativity case on all the data in our Fig. 2 is an unimpressive 2.51 [df 11]. In light of the concerns expressed above for the sensitivity of results on the large bias correction introduced in Ref. [3] we also checked if perhaps the non-tachyonic-special-relativity case could be rescued by allowing the estimate of the bias $b_{1979}$ given in Ref. [3] to vary within a 3 standard deviations range (standard deviation of $b_{1979}$ also estimated in Ref. [3], as quoted above). But by varying the bias parameter $b_{1979}$ accordingly we found that the reduced $\chi^{2}$ of the fit can only get worse. So we conclude that taking at face value the OPERA results and including them in such test of hypothesis would clearly disfavor standard non-tachyonic special relativity. 


\section{B. Superluminal but not a tachyon}

Clearly, if taken at face value, the data presently available point toward a superluminal $\mu$ neutrino. One could actually argue that all three results OPERA/CNGS11, MINOS/FERMILAB07 and FERMILAB79 individually favor (some more some less significantly) a $\mu$ neutrino with speeds greater than $c$. There is a common tendency to associate the concept of a "superluminal particle" (speed greater than the speed-of-light scale $c$ ) to a special-relativistic tachyon (particle governed by special relativity, but with imaginary mass). This is evidently not a correct association (and readers unfamiliar with the subject may use the illustrative examples of models here considered as guidance). But nonetheless we find appropriate to first test the hypothesis of the $\mu$ neutrino as a special-relativistic tachyon, as described by Eq. (4).

In Fig. 3 we show the fit of the special-relativistic-tachyon hypothesis on the data already summarized in our Fig. 2. We computed the reduced $\chi^{2}$ of this fit and found a discouraging 2.26 [df 10]. Also for this special-relativistic-tachyon hypothesis we then allowed the bias $b_{1979}$ to vary within its 3standard-deviation range, but could only find a negligible improvement in the reduced $\chi^{2}(2.25)$ in correspondence of a best-fit value for $\mathcal{M}^{2}$ of $\mathcal{M}^{2}=1.13 \cdot 10^{-3} \mathrm{GeV}^{2}$. From the high values of reduced $\chi^{2}$ we conclude that the specialrelativistic-tachyon hypothesis is disfavored ${ }^{4}$, even just using data on $\mu$ neutrinos in the energy range from 3 to $200 \mathrm{GeV}$.

Let us then warm up to the idea of a superluminal particle without imaginary mass, by considering the simplest option of the Coleman-Glashow scenario of Eq. (5). Fig. 4 shows the result of a fit of the Coleman-Glashow parameter on the OPERA+FERMILAB07+FERMILAB79 data already shown in our Fig. 2. The result is satisfactory, as implied by the reduced $\chi^{2}$ of the fit which we computed to be 1.26 [df 10]. Moreover, considering again values of $b_{1979}$ within its 3 -standard-deviation range, we found even lower values of reduced $\chi^{2}$ for the fit based on the Coleman-Glashow scenario, including a case with reduced $\chi^{2}$ of 0.70 in correspondence of a best-fit value of the Coleman-Glashow $\delta$ parameter of $\delta=2.6 \cdot 10^{-5}$.

So the Coleman-Glashow picture passes our test rather comfortably.

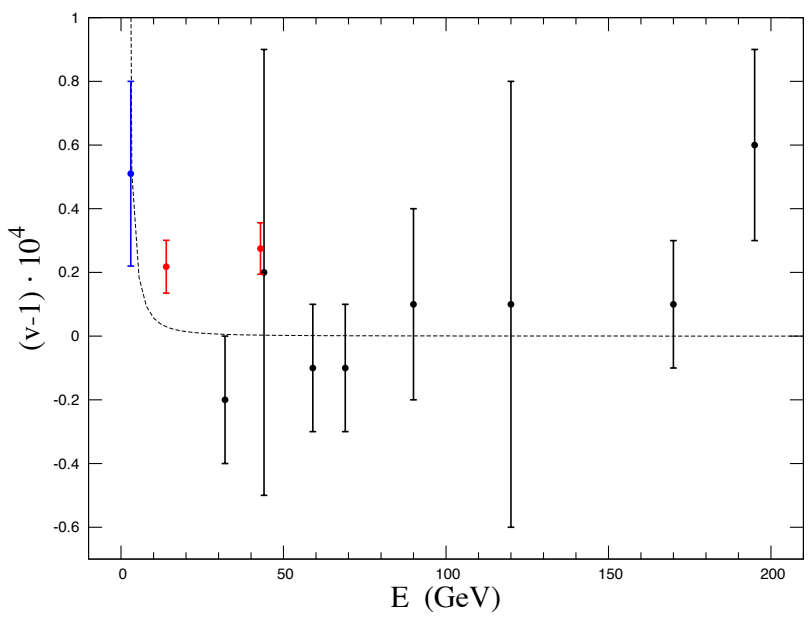

FIG. 3. Fit with the special-relativistic-tachyon hypothesis.

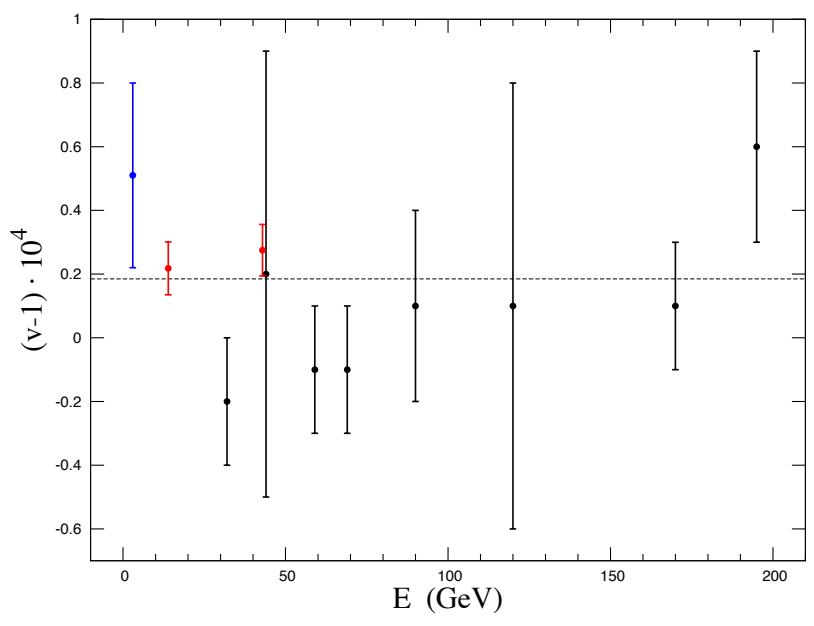

FIG. 4. Fit with the Coleman-Glashow hypothesis.

\footnotetext{
${ }^{4}$ Of course, it is a peculiar exercise to constrain a special-relativistic tachyon hypothesis using the more benign high-energy features rather than the pathological implications at lower energies. However, for reasons that will be stressed in the next section, there is some added value for us to constrain the special-relativistic tachyon with data at energies between a few $\mathrm{GeVs}$ and $200 \mathrm{GeVs}$.
} 


\section{The DSR-compatible cases}

As mentioned, the Coleman-Glashow scenario has only been studied and known to produce acceptable physics as a scenario for a full breakdown of special-relativistic Lorentz symmetry. Taking at face value the available data the only special-relativistic superluminal option, the tachyon, is "ruled out". From the previous subsection we do have a viable candidate, the Coleman-Glashow case but requires a preferred frame (an "ether frame"). Next let us explore another question: if one takes at face value the presently-available data is it then automatic that one is forced to violate the Relativity Principle and formulate the theory in an "ether frame"?

We here explore this by considering the DSR-compatible cases of Eq. (6) and of Eq. (7) for which it is established that the modification of the speed law can be implemented relativistically ${ }^{5}$

In Figs. 5 and 6 we show the results of fitting respectively the case of Eq. (6) and the case of Eq. (7) on the OPERA+FERMILAB07+FERMILAB79 data already shown in our Fig. 2. The results are not encouraging: those fits come with a reduced $\chi^{2}$ of 2.01 [df 10] for the case of Eq. (6), in Fig. 5, and of 2.35 [df 10] for the case of Eq. (7).

For the DSR-compatible quadratic case of Eq. (7) the outlook does not improve much even allowing again for varying the bias $b_{1979}$ within its 3-standard-deviation range: the best reduced $\chi^{2}$ we find following this procedure is is still of 2.06 (and would best-fit the parameter $\ell_{2}^{2}$ to the value $\ell_{2}^{2}=1.8 \cdot 10^{-9} \mathrm{GeV}^{-2}$ ). We find this value of reduced $\chi^{2}$ still not encouraging, and we therefore conclude that already with data available in the range from $3 \mathrm{GeV}$ to $200 \mathrm{GeV}$ the the DSR-compatible quadratic case of Eq. (7) appears to be disfavored.

For the DSR-compatible linear case of Eq. (6) the outlook appears to be more encouraging. By allowing the bias $b_{1979}$ to vary within its 3 -standard-deviation range one finds a sizable region with values of reduced $\chi^{2}$ close to 1 , including a case with reduced $\chi^{2}$ of 1.10 where the fitting value of the parameter $\ell_{1}$ is $\ell_{1}=3.96 \cdot 10^{-7} \mathrm{GeV}^{-1}$. We shall therefore consider the DSR-compatible linear case of Eq. (6) as a plausible description of the OPERA+FERMILAB07+FERMILAB79 on the speed of $\mu$ neutrinos.

\footnotetext{
${ }^{5}$ For completeness we note here (even though it is irrelevant for the narrow scopes of the present exploratory study) that just like deforming the Galilean boosts into Lorentz boosts requires the introduction of relativity of simultaneity, we recently understood [26-29] that in turn deforming Lorentz boosts into DSR-Lorentz boosts requires the introduction of relativity of locality.
}

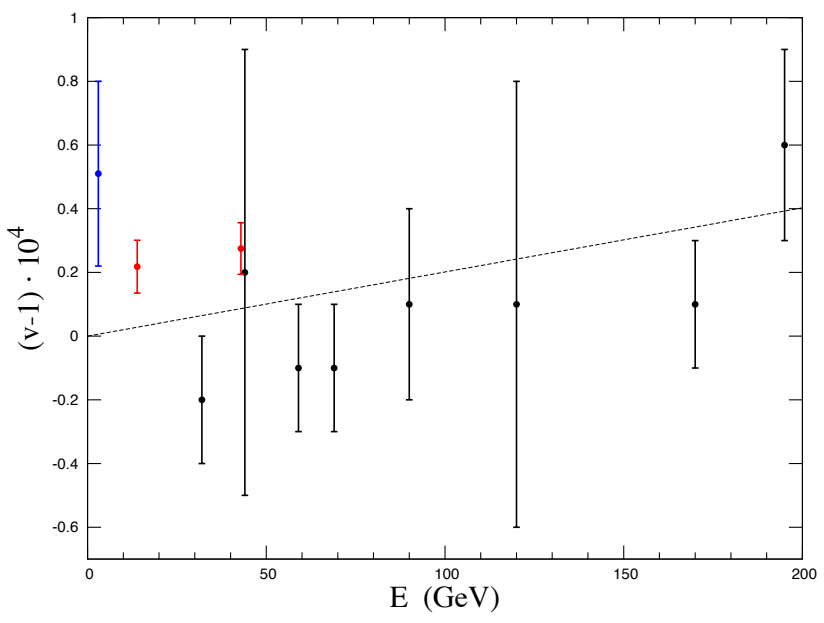

FIG. 5. Fit with the DSR-compatible linear case.

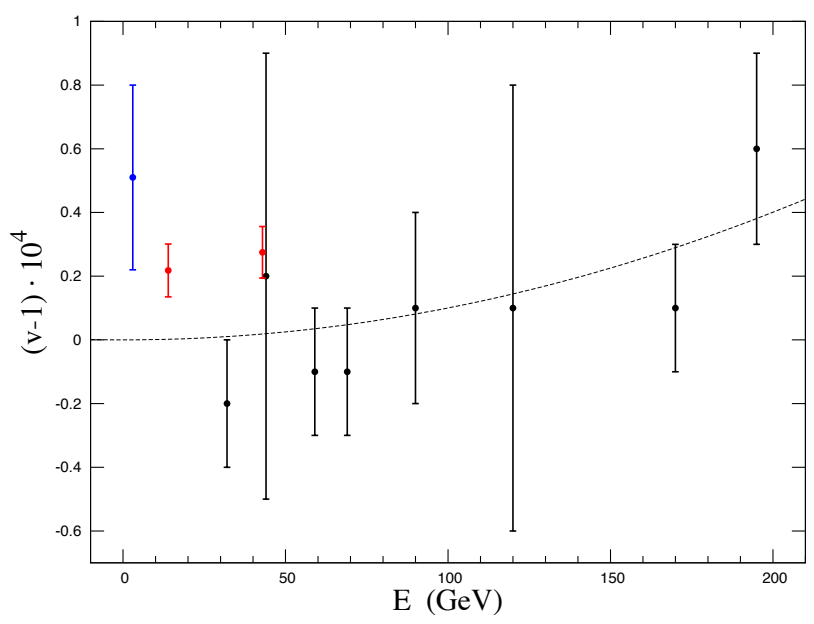

FIG. 6. Fit with the DSR-compatible quadratic case.

\section{SN1987A, OTHER NEUTRINO SPECIES AND LARGE EXTRA DIMENSIONS}

We have focused so far on a range of energies which is considerably wider than the OPERA range, but still very narrow in absolute terms. And we focused on data which apply (or can be interpreted as applying) exclusively to $\mu$ neutrinos. We shall soon argue that there are some advantages to this approach in a situation such as the one raised by the data recently reported by OPERA.

But before we do that let us instead widen our horizons, considering other neutrino species and other ranges of energy. From this perspective one should immediately consider the observations of neutrinos from the supernova 1987a (see, e.g., Refs. [30-32]). It is useful from this perspective to consider the Coleman-Glashow picture, which did very well in our peculiar test on the OPERA+FERMILAB07+FERMILAB79 data, and also the linear DSR-compatible case of Eq. (6), which in our analysis produced a plausible description of 
OPERA+FERMILAB07+FERMILAB79 data.

This happens to be also a good combination of cases on which to look at the SN1987a story, since it allows to illustrate the difference between an energy-independent modification of the speed law and an energy-dependent one.

Let us start with the energy-independent Coleman-Glashow case. If the same considerations we applied above to $\mu$ neutrinos in the range of 3 to $200 \mathrm{GeVs}$, are applied universally to neutrinos of any type and any energy then the ColemanGlashow picture, which provided a nice fit of $\mu$-neutrino data between $3 \mathrm{GeV}$ and $200 \mathrm{GeV}$, is immediately ruled out by the bound $|v-1|<2 \cdot 10^{-9}$ on neutrino speeds that one robustly infers [30, 31] from the observations of neutrinos from the supernova 1987a. In fact, our good fit to data in the $3-200 \mathrm{GeV}$ range with the Coleman-Glashow picture gave $v-1=\delta \sim 2.6 \cdot 10^{-5}$, which in the energy-independent Coleman-Glashow picture should be applicable to neutrinos of any energy, including the ones of SN1987a.

The situation is not equally disastrous for scenarios where the departures from special relativity increase with energy, such as the DSR-compatible case of Eq. 6. These scenarios predict effects for lower-energy SN1987a neutrinos which are of course weaker than the ones they predict for $\mathrm{GeV}$ neutrinos. So in principle one could find agreement between a larger effect at $\mathrm{GeV}$ energies and a smaller effect for SN1987a neutrinos. But the behavior must be very steep and one of the striking findings of the analysis we reported in the previous section is that, if one empowers the analysis with all the data available between $3 \mathrm{GeV}$ and $200 \mathrm{GeV}$ it emerges that the data situation favors energy independence or at most a softly increasing dependence on energy, not a very steep energy dependence such as needed for matching the large difference in magnitude between the feature reported by OPERA and the SN1987a bound.

And we should stress that looking at the prudent characterization of the bound obtainable from SN1987a neutrinos given, e.g., in Refs. [30, 31] one can very significantly underestimate how steep the dependence on energy would have to be in order to find agreement between the feature reported by OPERA and the SN1987a bound. The bound in Refs. [30, 31] very prudently took as reference the overall differences in times of arrival of SN1987a neutrinos and photons, but for a scenario with energy-dependent speed of neutrinos a much more severe constraint is obtained on the basis of the fact that SN1987a neutrinos of different energies reached the same detectors within a few seconds.

Let us illustrate this issue considering a generic power-law energy dependence

$$
v-1=\left(\frac{E}{M_{*}}\right)^{\alpha}
$$

for some scale $M_{*}$ and some power $\alpha$. If one imposes on this ansatz the constraint that Kamiokande observed SN1987a neutrinos $7.5 \mathrm{MeV}$ and a $35 \mathrm{MeV}$ within 13 seconds of each other and if one also imposes $v-1 \sim 2 \cdot 10^{-5}$ at $20 \mathrm{GeV}$ (as a rough characterization of the result reported by OPERA) the conclusion is that all $\alpha<2.5$ appear to be disfavored.

In a companion paper now in preparation [20] we shall establish that no simple functional dependence on energy can successfully satisfy both the demands of our approach using data from 3 to $200 \mathrm{GeV}$ on $\mu$ neutrinos and the constraints contained in the SN1987a story.

We feel that if the OPERA result is taken at face value then its description cannot be a simple mechanism based on a universal energy-speed relation with familiarly smooth functional form. We expect it to be necessary to advocate strong neutrino-flavor dependence of the effect and/or some mechanism that switches on the superluminal behavior only at energies higher that the SN1987a energies. And while such peculiar specifications of models are evidently unappealing, it should be noticed that a strong flavor dependence of Lorentzviolation effects is for example not foreign to some frontier area of research, such as spacetime noncommutativity and other areas of quantum-gravity research. Even more noteworthy is the status of onset scales within the large-extradimension literature. As mentioned studies from more than a decade ago [15, 16] suggested that large extra dimensions should lead to superluminal behavior of neutrinos with an onset scale, below which the effect would be completely switched off. The fact that such theoretical speculations where put forward well before the recent interest in superluminal neutrinos must be viewed as an element of compellingness. And more recently (but still much before the OPERA study) other large-extra-dimension investigations have reported the possibility of superluminal behaviour for neutrinos, confined to specific ranges of energy [18, 19].

It is in light of these considerations that we expect that ultimately the main arena for scrutinizing the intriguing OPERA result will have to be the context of studies of the velocity of $\mu$ neutrinos in the energy range between a few GeVs and, say, 200 or $300 \mathrm{GeVs}$. And it is in preparation for this task that we set up in the previous section the richest analysis of this sort that could be done on presently available data.

\section{CLOSING REMARKS}

We have shown that combining all data presently available for $\mu$ neutrinos in the energy range from $3 \mathrm{GeV}$ to 200 $\mathrm{GeV}$ one achieves tangible discriminating power for candidate models of a superluminal $\mu$ neutrino.

And we have argued, at the end of the previous section, that ultimately the main arena for scrutinizing the intriguing OPERA result will have to be the context of studies of the velocity of $\mu$ neutrinos in the energy range between a few GeVs and, say, 200 or $300 \mathrm{GeVs}$.

We feel that a priority for future experiments should be involving the $\mu$ neutrinos of the highest energies manageable, since this would further empower the strategy of analysis we here proposed.

Evidently also relying on experiments with significantly different baselines would be a major asset for the discrimination of a propagation effect with respect to several candidate sources of systematic bias for velocity measurements.

GG thanks for the hospitality the APC - Universite Paris 7, where part of this work was carried out. 
[1] T. Adam et al [OPERA collaboration], arXiv:1109.4897v1

[2] P. Adamson et al. [ MINOS Collaboration ], Phys. Rev. D76, 072005 (2007). arXiv:0706.0437 [hep-ex]].

[3] G. R. Kalbfleisch, N. Baggett, E. C. Fowler and J. Alspector, Phys. Rev. Lett. 43, 1361 (1979)

[4] S. R. Coleman, S. L. Glashow, Phys. Lett. B405, (1997) 249252. |hep-ph/9703240|.

[5] S. R. Coleman, S. L. Glashow, Phys. Rev. D59 (1999) 116008. |hep-ph/9812418|.

[6] G. Amelino-Camelia, arXiv:gr-qc/0207049, Nature 418 (2002) 34-35.

[7] G. Amelino-Camelia, arXiv:gr-qc/0012051 Int. J. Mod. Phys. D11 (2002) 35.

[8] G. Amelino-Camelia, arXiv:hep-th/0012238, Phys. Lett. B 510 (2001) 255.

[9] J. Kowalski-Glikman, arXiv:hep-th/0102098 Phys. Lett. A 286 (2001) 391.

[10] J. Magueijo and L. Smolin, arXiv:hep-th/0112090 Phys. Rev. Lett. 88 (2002) 190403.

[11] G. Amelino-Camelia, J. Ellis, N.E. Mavromatos, D.V. Nanopoulos and S. Sarkar, arXiv:astro-ph/9712103, Nature 393 (1998) 763-765.

[12] R. Gambini and J. Pullin, Phys. Rev. D59 (1999) 124021.

[13] J. Alfaro, H.A. Morales-Tecotl and L.F. Urrutia, gr-qc/9909079 Phys. Rev. Lett. 84 (2000) 2318.

[14] L. Smolin, Lect. Notes Phys. 669 (2005) 363.

[15] V. Ammosov, G. Volkov, arXiv:hep-ph/0008032

[16] G. G. Volkov, hep-ph/0607334, Annales Fond. Broglie 31 (2006) 227.
[17] A. de Gouvea, Gian F. Giudice, A. Strumia, K. Tobe, Nucl.Phys.B623 (2002) 395, arXiv:hep-ph/0107156

[18] J. Dent, H. Päs, S. Pakvasa, T. J. Weiler, arXiv:0710.2524

[19] S. Hollenberg, H. Päs arXiv:0904.2167

[20] G. Amelino-Camelia, G. Gubitosi, P. Lipari, N. Loret, F. Mercati and G. Rosati, in preparation

[21] V.A. Kostelecky and C.D. Lane, Phys. Rev. D60 (1999) 116010.

[22] V.A. Kostelecky and N. Russell, arXiv:0801.0287. Rev.Mod.Phys. 83 (2011) 11

[23] J. Albert et al. [MAGIC Collaboration] and J.R. Ellis, N.E. Mavromatos, D.V. Nanopoulos, A.S. Sakharov and E.K.G. Sarkisyan, Phys. Lett. B 668 (2008) 253.

[24] G. Amelino-Camelia and L. Smolin, arXiv:0906.3731 [astroph.HE], Phys. Rev. D80 (2009) 084017.

[25] J. R. Ellis, N. Harries, A. Meregaglia, A. Rubbia, A. Sakharov, Phys. Rev. D78 (2008) 033013. [arXiv:0805.0253 [hep-ph]].

[26] G. Amelino-Camelia, M. Matassa, F. Mercati and G. Rosati, arXiv:1006.2126 Phys. Rev. Lett. 106, 071301 (2011).

[27] L. Smolin, arXiv:1007.0718

[28] G. Amelino-Camelia, L. Freidel, J. Kowalski-Glikman L. Smolin, arXiv:1101.0931

[29] G. Amelino-Camelia, L. Freidel, J. Kowalski-Glikman L. Smolin, arXiv:1106.0313, Gen. Relativ. Gravit. 43 (2011) 2547.

[30] L. Stodolsky Pys. Lett. B 201 pp. 353, (1988)

[31] M. J. Longo, Phys. Rev. D 36, 3276 (1987).

[32] P. Galeotti and G. Pizzella, arXiv:0706.2235 (presented at the XII-th International Workshop on Neutrino Telescope, Venice, 6-9 March 2007) 